NASATM- - $\varepsilon: /-207540$

PLPNT

フリーンターナク

475

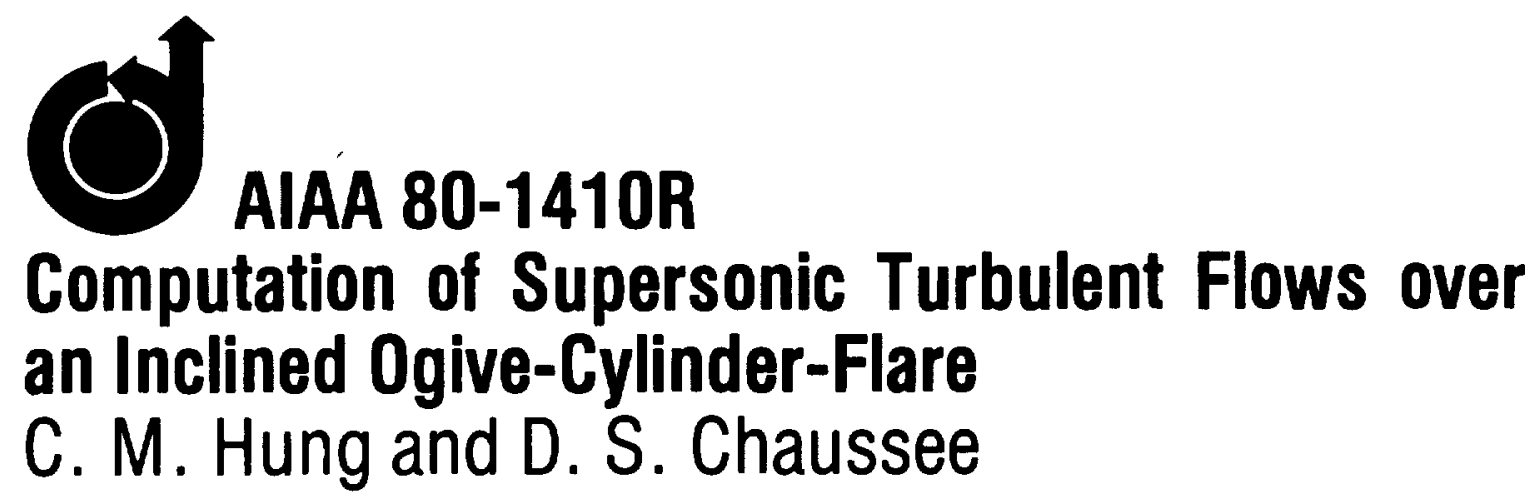




\title{
Computation of Supersonic Turbulent Flows over an Inclined Ogive-Cylinder-Flare
}

\author{
C. M. Hung * \\ NASA Ames Research Center, Moffett Field, Calif. \\ and \\ D. S. Chaussee $\dagger$ \\ Flow Simulations, Inc., Sunnyvale, Calif.
}

\begin{abstract}
A supersonic turbulent flow over an ogive-cylinder-flare has been solved numerically. The calculations proceed in two parts. Initially, the parabolized Navier-Stokes equations are solved for the ogive cylinder back to a location upstream of the shock-wave and boundary-layer inieraction. Then, the time-dependent Navier-Stakes equations with a thin-layer approximation are solved for the remaining cylinder-flare portion. Results for a Mach number of 2.0 and a unit Reynolds number of $11.42 \times 10^{6} / \mathrm{m}$ are obtained for angles of atiack $\alpha=0,4$, and $8 \mathrm{deg}$. Good agreement has been found between computed and experimental results of the surface pressure on the ogive-cylinder portion, and for the interaction region at $\alpha=0$ and 4 deg. The role of circumferential communication in a three-dimensional shock-wave and boundary-layer interaction fowfield is discussed.
\end{abstract}

\section{Introduction}

A $N$ ogive-cylinder-flare configuration (Fig. 1) is commonly seen in rockets, missiles, and space launch vehicles. The ogive-cylinder is a basic slender aerodynamic body, with the flare providing a natural fairing for multistage spacecraft. In many circumstances, the flared afterbody is also used to stabilize bodies in flight or to simulate underexpanded rocket plumes. With advances in computers and numerical techniques, inviscid supersonic calculations over such a body are well developed and can be obtained in seconds or minutes of computation time. ${ }^{1}$ However, in reality, a fluid is viscous, and it is necessary to take into account the viscous effects in order to predict the total force and moments accurately.

Two interesting features are associated with viscous effects on a slender body with a flare. The first and the most common feature is the lee-side crossflow separation that strongly affects the lift, drag, heating, and stability of a slender body, especially at large angles of attack. The second feature is the shock-wave and boundary-layer interaction near the corner of the frustum (or flare). The pressure rise due to the "flare" shock induces flow recirculation near the corner. The variation of shock strength and the crossflow in the meridional direction make the interaction flowfield three dimensional. Moreover, the existence of lee-side separation enriches the three-dimensional feature further, as illustrated in Fig. 2, with oil-flow experiments by Chyu and $\mathrm{Coe}^{2}$ on a cylinder with a $23 \mathrm{deg}$ cone frustum for various angles of attack.

Computation of viscous supersonic flows over a body of revolution and a conical body without streamwise separation have been made previously with varying degrees of success (e.g., Refs. 3-8). Among them, the recently developed parabolized Navier-Stokes code using an implicit numerical technique' appears to be very promising for use as a research and design tool.

Presented as Paper $80-1410$ at the AIAA 13th Fluid and Plasma Dynamics Conference, Snowmass, Colo., July 14-16, 1980; submitted Sept. 23, 1980; revision received March 16, 1981. This paper is declared a work of the U.S. Government and therefore is in the public domain.

- Research Scientist, Computational Fluid Dynamics Branch. Member AlAA.

†Senior Research Scientist. Member AIAA.
Hung ${ }^{9}$ solved the time-dependent, thin-layer approximation of the Navier-Stokes equations for a supersonic laminar flow over a hollow cylinder with a flare at angles of attack. The numerical results show good agreement with experimental measurement of surface pressure and normal force distribution. The results also show that the circumferential communication by means of the crossflow plays an important role in this three-dimensional shockwave/boundary-layer interaction flowfield. In the present study, the previous method' ${ }^{9}$ is extended to high Reynolds number turbulent flow case.

The objectives of the present investigation are 1) to demonstrate the feasibility of computing supersonic turbulent flow over an ogive-cylinder-frustum, 2) to study the associated three-dimensional shock-wave/turbulent boundary-layer interaction near the cylinder-flare juncture, and 3) to assess the role of crossflow separation in this interaction flowfield.

To efficiently solve the problem, the calculations are done in two parts. Initially, the parabolized Navier-Stokes equations (Ref. 7, also employing the thin-layer approximations) are solved using an implicit factored scheme ${ }^{10}$ for the portion of the ogive-cylinder up to a location $x_{0}$, which is ahead of the cylinder-flare interaction region. Then, the time-dependent thin-layer Navier-Stokes equations (Ref. 9 , with extension to turbulent flow) are solved for the remaining cylinder-flare portion where the streamwise recirculation occurs. The time-dependent Navier-Stokes equations are solved using a mixed explicit-implicit scheme developed by MacCormack." Since the parabolized equations are solved by matching a data plane in the streamwise direction, the required computation time and storage are substantially less than those required for the time-dependent approach. However, the time-dependent approach is needed to account for the shock-wave and boundary-layer interaction since the parabolized equations are not stable in the region of streamwise separated flows.

The calculated results are compared with the experimental data of Ref. 2 at Mach number 2.0, unit Reynolds number $11.42 \times 10^{6} / \mathrm{m}\left(0.29 \times 10^{6} / \mathrm{in}.\right)$, and angles of attack $\alpha=0,4$, and 8 deg. Inviscid calculations are also presented for the cylinder-frustum portion to show the viscous effects. Since the employed parabolized Navier-Stokes approach has been previously documented, ${ }^{7,8}$ this paper will have more emphasis on the development and the results of the time-dependent calculation. 


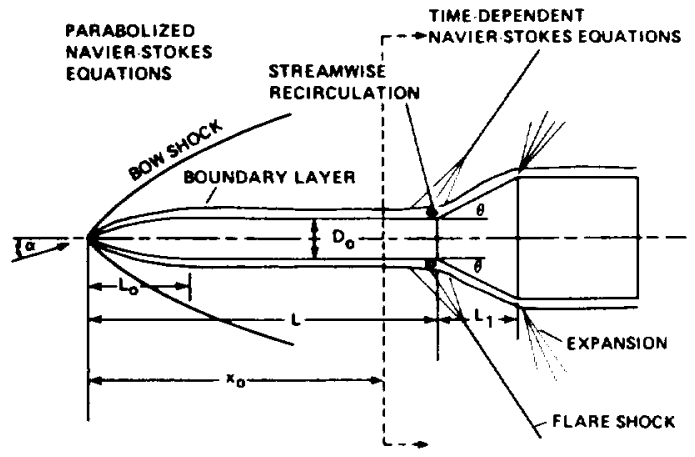

Fig. 1 Supersonic flows over an ogive-cylinder-flare.
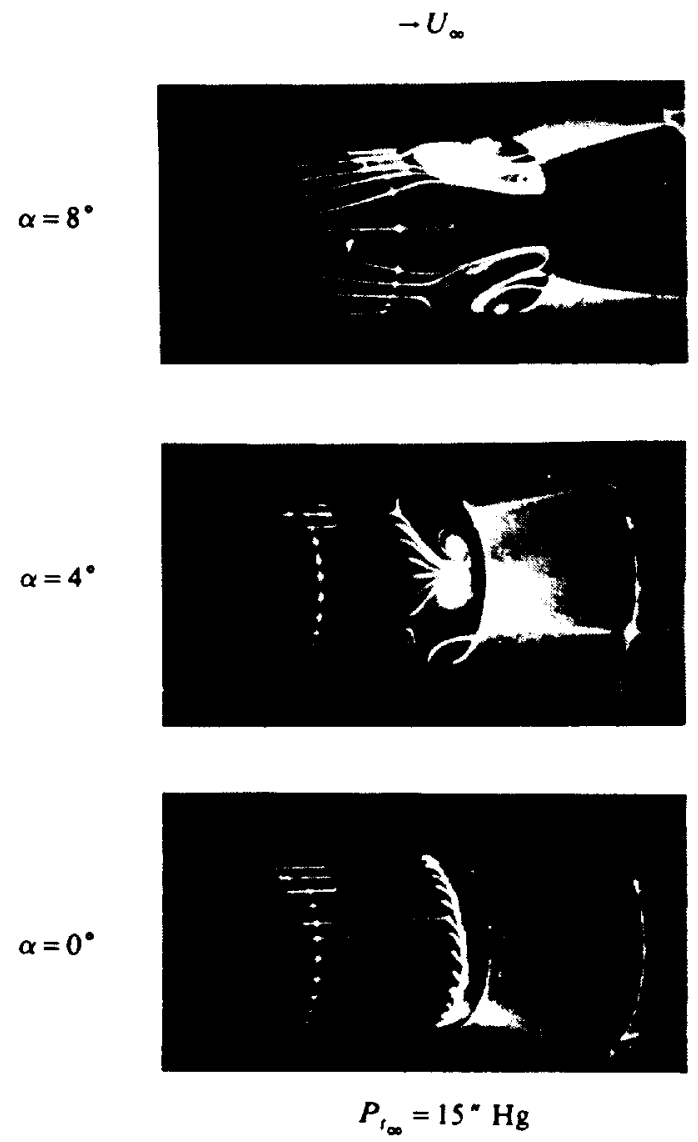

Fig. 2 Fluorescent oil studies of flow on a 10-in.-diam model with a 23-deg cone frustum; $M_{\infty}=2.0$.

\section{Analysis}

Thin-Layer Approximation

Figures $3 \mathrm{a}$ and $3 \mathrm{~b}$ show cross-sectional views of the computational domain in the $(x, r)$ and $(r, \phi)$ planes for a cylinder frustum. Here, $\theta$ is the cone half-angle of the flare. The flow is assumed to be symmetric in $\phi$, and hence only the half-plane 0 deg $\leq \phi \leq 180 \mathrm{deg}$ is needed. The mesh is uniformly spaced in both the $x$ and $\phi$ directions. In the $r$ direction, a fine-mesh spacing is used in the region near the body $r_{b} \leq r \leq r_{\text {, }}$ to resolve the viscous forces, and a coarsemesh spacing is used in the outer region $r_{f}<r<r_{0}$, where viscous effects are negligible. Both fine and coarse meshes are geometrically stretched.

The governing equations of the present analysis for the cylinder-frustum region are the time-dependent, com-

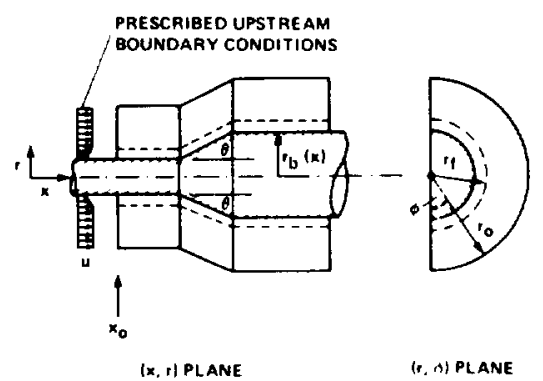

Fig. 3 Cross sections of the computational domain.

pressible, Navier-Stokes equations, cast in terms of massaveraged variables, with the bulk viscosity and specific turbulent energy in the normal stress components omitted. The resulting mean conservative relations are the same as their laminar flow counterparts, except for the addition of the Reynolds stress tensor and the Reynolds heat flux. Turbulent closure is accomplished by expressing the Reynolds stress tensor in terms of the product of an eddy viscosity $\epsilon$ with the mean velocity gradient. Also, a turbulent Prandtl number $\boldsymbol{P r}$, is used for the Reynolds heat flux. In high Reynolds number flows, however, the viscous effects are confined near the wall boundary and are dominated by the viscous terms associated with the derivatives in the direction outward from the wall. Consequently, all viscous terms associated with derivatives along the body are neglected, while those with second-order derivatives in the direction outward from the body are retained. Written in weak conservative form in transformed cylindrical coordinates, the thin-layer approximate NavierStokes equations are as follows.

$$
\frac{\partial r U}{\partial t}+\frac{\partial r F}{\partial \xi}+\frac{\partial r\left(G-G_{v}\right)}{\partial \eta}+\frac{\partial r H}{r \partial \phi}+Q=0
$$

where

$$
\begin{gathered}
\xi=x, \quad \eta=r-r_{b}(x), \quad v^{\prime}=v-u r_{b}^{\prime}(x) \\
r_{b}^{\prime}(x)=0 \text { for } x \leq L \text { or } x>L+L_{l} \\
=\tan \theta \text { for } L<x<L+L_{I}
\end{gathered}
$$$$
U=\left[\begin{array}{c}
\rho \\
\rho u \\
\rho v \\
\rho w \\
\rho E
\end{array}\right] F=\left[\begin{array}{c}
\rho u \\
\rho u^{2}+p \\
\rho v u \\
\rho w u \\
(\rho E+p) u
\end{array}\right] G=\left[\begin{array}{c}
\rho v^{\prime} \\
\rho u v^{\prime}-p r_{b}^{\prime}(x) \\
\rho v v^{\prime}+p \\
\rho w v^{\prime} \\
(\rho E+p) v^{\prime}
\end{array}\right]
$$$$
H=\left[\begin{array}{c}
\rho w \\
\rho u w \\
\rho v w \\
\rho w^{2}+p \\
(\rho E+p) w
\end{array}\right] Q=\left[\begin{array}{c}
0 \\
0 \\
-p-\rho w^{2}+\sigma_{\phi \phi} \\
+\rho v w-\sigma_{r \phi} \\
0
\end{array}\right]
$$

$$
G_{v}=\left[\begin{array}{c}
0 \\
\sigma_{v x}-r_{b}^{\prime}(x) \sigma_{x x} \\
\sigma_{v r}-r_{b}^{\prime}(x) \sigma_{r x} \\
\sigma_{r \phi}-r_{b}^{\prime}(x) \sigma_{\phi x} \\
\Phi_{,}-r_{b}^{\prime}(x) \Phi_{x}
\end{array}\right]
$$




$$
\begin{aligned}
& \Phi_{x}=\sigma_{x x} u+\sigma_{r x} v+\sigma_{\phi x} w-\left(\frac{\gamma \mu}{P r}+\frac{\gamma \epsilon}{P r_{t}}\right) \frac{\partial e_{i}}{\partial \eta} r_{b}^{\prime}(x) \\
& \Phi_{r}=\sigma_{r x} u+\sigma_{r r} v+\sigma_{r \phi} w+\left(\frac{\gamma \mu}{P r}+\frac{\gamma \epsilon}{P r_{t}}\right) \frac{\partial e_{i}}{\partial \eta} \\
& \sigma_{x x}=\lambda \frac{\partial}{\partial \eta}\left[v-u r_{b}^{\prime}(x)\right]-2(\mu+\epsilon) \frac{\partial u}{\partial \eta} r_{b}^{\prime}(x) \\
& \sigma_{r r}=\lambda \frac{\partial}{\partial \eta}\left[v-u r_{b}^{\prime}(x)\right]+2(\mu+\epsilon) \frac{\partial v}{\partial \eta} \\
& \sigma_{\phi \phi}=\lambda \frac{\partial}{\partial \eta}\left[v-u r_{b}^{\prime}(x)\right] \quad \sigma_{r x}=(\mu+\epsilon) \frac{\partial}{\partial \eta}\left[u-v r_{b}^{\prime}(x)\right] \\
& \sigma_{r \phi}=(\mu+\epsilon) \frac{\partial}{\partial \eta} w \quad \sigma_{\phi x}=-(\mu+\epsilon) \frac{\partial}{\partial \eta} w r_{b}^{\prime}(x)
\end{aligned}
$$

Here, $L$ is the distance from the ogive nose to the fare juncture and $L$, the length of the flare portion, as described in Fig. 1 ; $\lambda=-2 / 3(\mu+\epsilon) ;(u, v, w)$ are velocity components in the axial, radial, and azimuthal $(x, r, \phi)$ directions; $\rho, p$, and $E$ are density, pressure, and total energy, with $E=e_{1}+0.5$ $\left(u^{2}+v^{2}+w^{2}\right)$, where $e_{i}$ is the specific internal energy. The perfect gas relation is $p=(\gamma-1) p e_{i}$. The molecular viscosity $\mu$ is evaluated by Sutherland's formula.

Note that the retention of all the second derivatives associated with the $\eta$ direction in Eq. (1) by no means implies that every retained viscous term is larger than those neglected. For instance, as seen from a boundary-layer analysis, the term $\partial v / \partial \eta$ in $\sigma_{x x}$ is of the same order of magnitude as the neglected term $\partial u / \partial \xi$. The retention of all (and just) the second derivatives with respect to the direction outward from the body is for consistency and convenience in extending the numerical code to a general geometry. However, as the name implies, the thin-layer approximation is valid only for problems where the viscous forces are confined to a thin layer. Another point is that, near the streamwise separation point, $\partial^{2} u / \partial \xi^{2}$ may be larger than $\partial^{2} u / \partial \eta^{2}$. However, this effect is localized (two or three points around the separation point), and the fluid has very low momentum near that point. Hence, it is believed that the thin-layer approximation is able to predict the general features of a flow with streamwise separation insofar as the separation is "small" and confined near the wall.

\section{Boundary Conditions}

The coordinates are body oriented, with the upstream boundary positioned at $x_{0}$ ahead of the shock-wave and boundary-layer interaction. The flow conditions at $x_{0}$ are prescribed from the results of the parabolized Navier-Stokes calculation. The outer boundary is set inside the bow shock generated from the ogive nose and outside the shock generated from the flare. In the present study, the ogivecylinder portion is long enough so that the outer boundary conditions are assumed uniform in the $\xi$ direction and are equal to the flow conditions at the outer edge of the upstream boundary. Note that the angle of attack is imposed implicitly through the upstream and the outer boundary conditions. Therefore, the accuracy of the upstream (and hence the outer) boundary conditions from the parabolized calculation-for both viscous and the inviscid portions-is important to the time-dependent calculation.

The symmetry condition is applied at $\phi=0$ - and $180-\mathrm{deg}$ planes. The wall is assumed impermeable, and a no-slip boundary condition is used. The wall is treated either as isothermal or as adiabatic, with the wall pressure being evaluated by the condition

$$
\partial p / \partial \eta=0 \text { at } \eta=0
$$

In the present cases, the first mesh point is so close to the wall that this pressure condition is appropriate. Details of the numerical procedures are described in Refs. 9 and 11.

\section{Eddy-Viscosily Model}

A two-layer turbulence model, developed by Baldwin and Lomax, ${ }^{12}$ is applied in the following fashion. In the inner region, the Prandtl mixing length is used

$$
\epsilon_{\text {inner }}=\rho(K D \eta)^{2} \omega
$$

where $D$ is the Van Driest damping factor, $K$ von Kármán's constant, and $\omega$ the absolute magnitude of vorticity, $\omega=|\nabla \times v|$. In the outer region,

$$
\epsilon_{\text {outer }}=C_{c p}\left(0.0168 \rho F_{\text {wake }} \beta\right)
$$

where

$$
F_{\text {wake }}=\left[\eta_{\max } F_{\max } \text { or } C_{w k} \eta_{\max } u_{\mathrm{e}}^{2} / F_{\max } ; \text { the smaller }\right]
$$

$F_{\max }$ is the maximum value of

$$
F(\eta)=\eta \omega D
$$

and $\eta_{\max }$ is the value of $\eta$ at which it occurs. The Klebanoff intermittency factor $\beta$ is given by

$$
\beta=\left[1+5.5\left(C_{\mathrm{Kleb}} \eta / \eta_{\max }\right)^{6}\right]^{-1}
$$

The quantity $u_{e}$ is the maximum total velocity at a fixed $(x, \phi)$ location. The constants appearing in the preceding relations are

$$
C_{c p}=1.6, \quad C_{w k}=0.25, \quad C_{\mathrm{Kleb}}=0.3
$$

In general, with no streamwise separation, $F_{\text {wake }}=$ $\eta_{\max } F_{\max }$. The eddy-viscosity model employed is similar to the Cebeci-Smith model ${ }^{13}$ and avoids the trouble of finding the edge of the boundary layer, which is difficult for nonuniform inviscid flows. [A small difference in defining $u_{e}$ can lead to a large difference in calculation of displacement thickness $\delta^{*}=\int_{\delta}^{\delta}\left(1-u / u_{e}\right) d \eta$.] Note, however, that in a streamwise separation region, very often the term $C_{w k} \eta_{\max } w_{e}^{2} /$ $F_{\max }<\eta_{\max } F_{\max }$, and hence the outer eddy viscosity may differ from the Cebeci-Smith model substantially.

\section{Results and Discussions}

The experiments selected for comparison were conducted by Chyu and Coe. ${ }^{2}$ The dimensions of the tested model are that the diameter of cylindrical portion $D_{0}=25.4 \mathrm{~cm}$ (10 in.), the ogive portion $L_{0}=3 D_{0}, L=7 D_{0}, L_{I}=17.95 \mathrm{~cm}$, and $\theta=23 \mathrm{deg}$. The flow conditions are freestream Mach number, $M_{\infty}=2.0$, Reynolds number based on $L, R e_{L}=20.3 \times 10^{6}$, with angles of attack $\alpha=0,4$, and $8 \mathrm{deg}$. The wall is assumed to be adiabatic. The calculations are done in two parts using different computer programs: first, a parabolized NavierStokes code for the ogive-cylinder portion, and then the timedependent approach for the remaining cylinder-frustum portion.

\section{Ogive Cylinder}

The parabolized Navier-Stokes code (PNS), developed by Schiff and Steger, ' is modified to solve the ogive-cylinder portion up to $x_{0} / D_{0}=6$. The PNS code also uses the thinlayer approximation and the Baldwin-Lomax two-layer turbulence model. To start the calculation for each angle of attack, a viscous "conical" solution is generated by the program at $x / D_{0}=0.1$, with a cone half-angle matching the body slope at that location. The solution is then marched in the axial $x$ direction with a constant step size. 40 grid points 

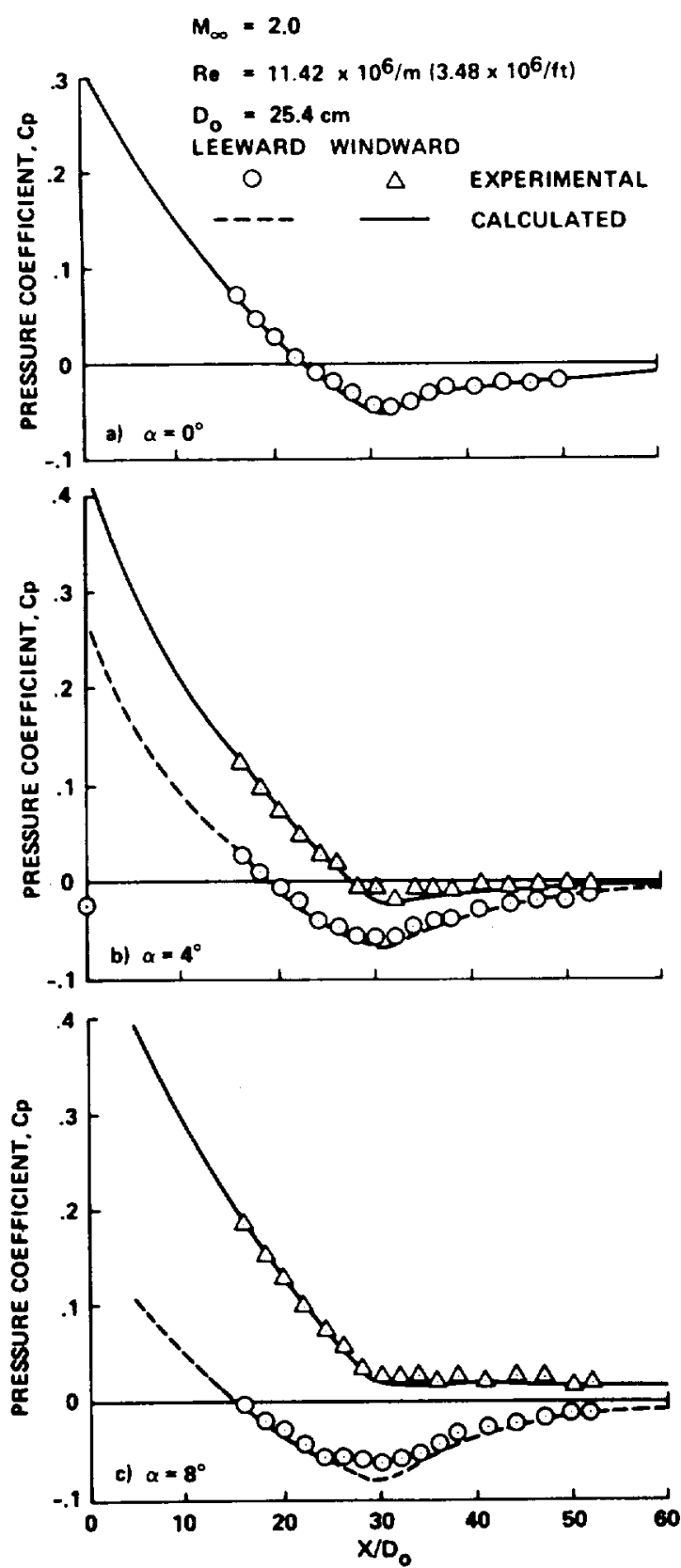

Fig. 4 Computed and experimental pressure coefficient on ogivecylinder.

are distributed in the $\eta$ direction, using a geometric stretching to ensure sufficient resolution in the laminar sublayer. $19 \mathrm{grid}$ points are evenly distributed on the meridional direction.

Figures $4 a-4 c$ compare the computed results from the parabolized Navier-Stokes code with experimental measurements of the surface pressure for three angles of attack, $\alpha=0,4$, and $8 \mathrm{deg}$. The agreement is good.

\section{Cylinder Frustum}

The computed results from the parabolized Navier-Stokes code at $x_{0} / D_{0}=6$ are interpolated to obtain the upstream boundary condition for the time-dependent approach. A grid of $45 \times 34 \times 20$ points encloses the following computational domain: $\xi / D_{0}$ from 6.0 to $8.04 ; \eta / D_{0}$ from 0 to $1.15 ; \phi$ from 0 to 180 deg. A geometrically stretched fine mesh (20 points) is used in the $\eta$ direction, from the body to $\eta / D_{a}=0.096$. A minimum-mesh cell spacing, $(\Delta \eta)_{\min }=0.12 L / \sqrt{R_{L}}$, results

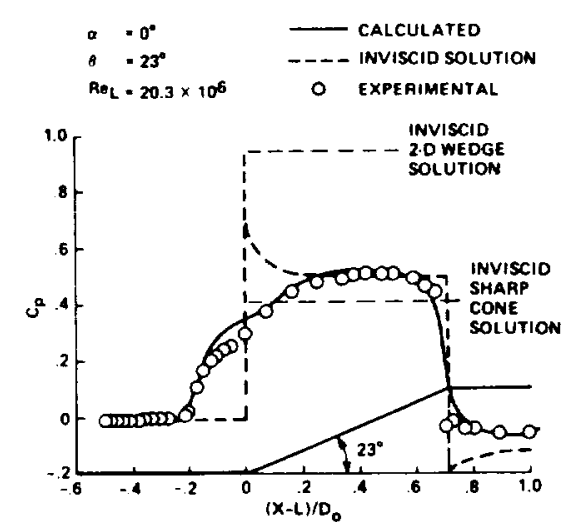

Fig. 5 Comparison of surface pressure at $\alpha=0$ deg.

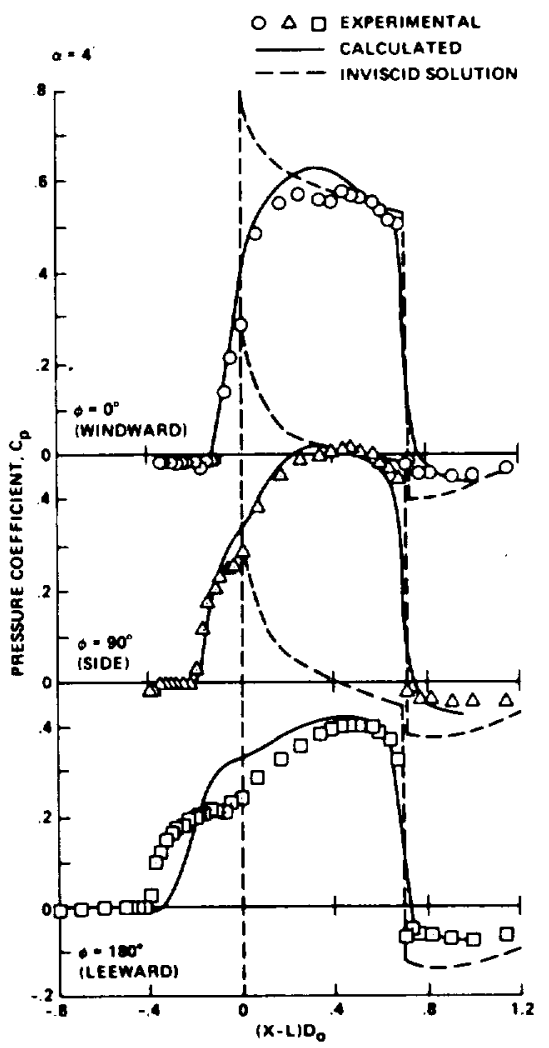

Fig. 6 Comparison of surface pressure at $\alpha=4$ deg.

in the first mesh point away from the body being located at about

$$
\eta^{+}=\eta\left[\sqrt{\frac{\rho(\partial u / \partial \eta)}{\mu}}\right]_{\text {wall }}=4
$$

Figure $S$ compares surface pressure at zero angle of attack. The agreement is good. Also shown in the figure are the inviscid solutions for a sharp cone, a two-dimensional wedge, and a cylinder frustum. Figures 6 and 7 compare surface pressure for $\phi=0,90$ and $180 \mathrm{deg}$ at angles of attack $\alpha=4$ and 8 deg. In Fig. 6, the agreement is good and the general features are predicted, except for the upstream pressure rise and hence streamwise separation point at $\phi=180 \mathrm{deg}$. At $\alpha=8 \mathrm{deg}$, the computed result for the windward plane $\phi=0$ deg still shows very good agreement with the experimental measurement. On the lee side, the viscous computation fails to produce a sharp pressure peak near the cylinder-frustum 


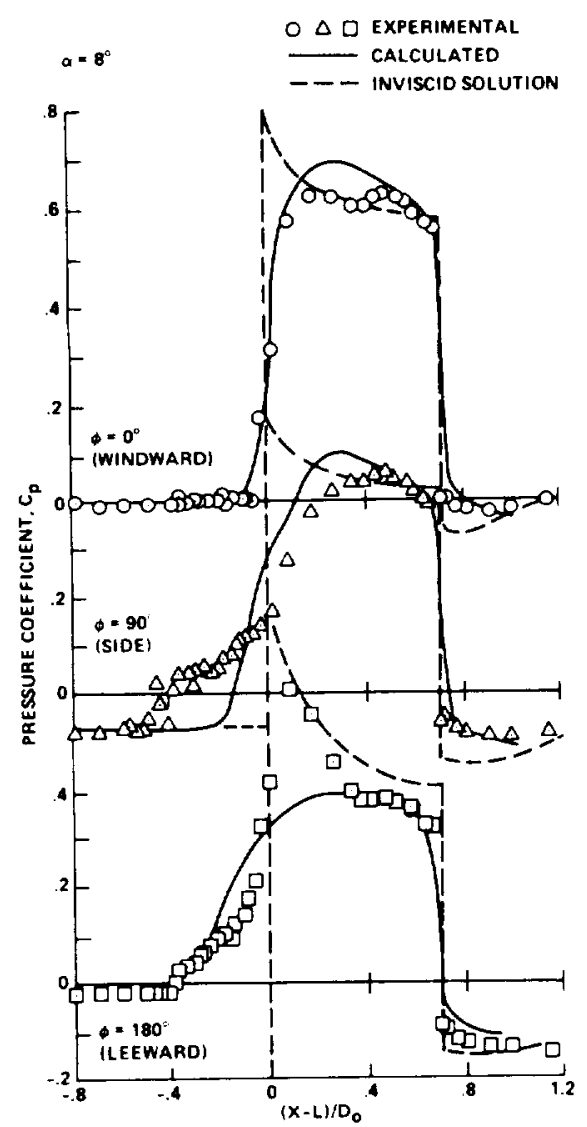

Fig. 7 Comparison of surface pressure al $\alpha=8 \mathrm{deg}$.

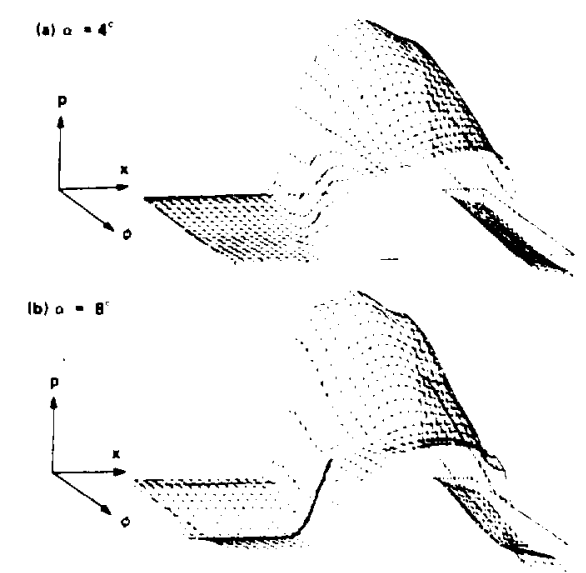

Fig. 8 Plots of surface pressure on $(x-\phi)$ plane.

juncture. (This will be discussed later.) The features of the surface pressure variation along a constant $\xi$, and a constant $\phi$ coordinate can be seen in Figs. $8 a$ and 8 b for $\alpha=4$ and 8 deg.

Plots of skin-friction coefficients in circumferential and streamwise directions $C_{f_{\phi}}$ and $C_{f x}$ are shown in Figs. 9a and $9 \mathrm{~b}$, for $\alpha=8 \mathrm{deg}$. A peak in $C_{f x}$ results from the expansion and acceleration of now around the convex frustum corner. In the circumferential direction, the maximum $C_{f \phi}$ shows around $\phi=90 \mathrm{deg}$, due to the strong crossflow. While no drastic change is shown near the convex corner, the $C_{f \phi}$ also shows a sharp decrease near the cylinder-flare juncture, associated with the streamwise separation, and becomes negative, even at a small angle of attack, $\alpha=4 \mathrm{deg}$ (not shown).

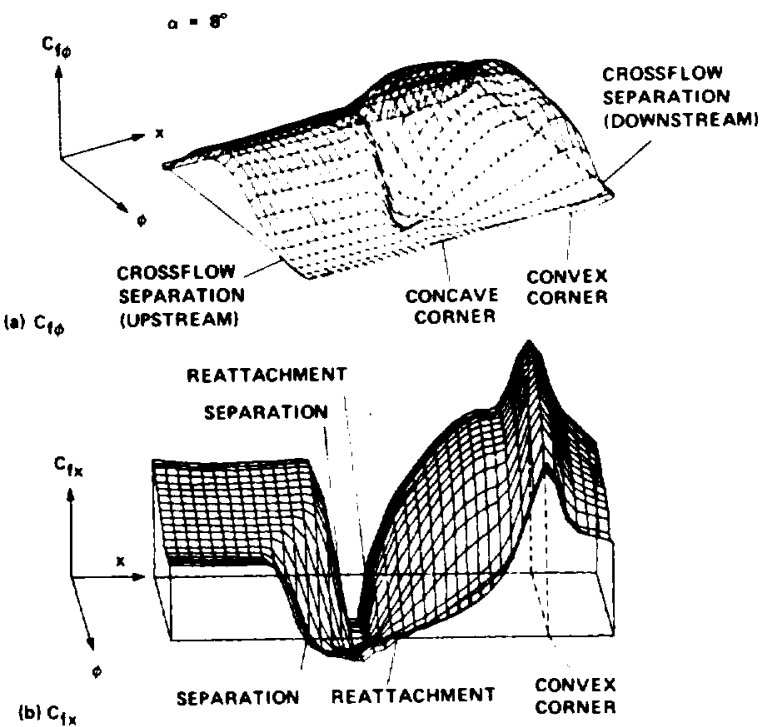

Fig. 9 Plots of skin friction coefficienl in circumferential and streamwise directions $C_{/ \phi}$ and $C_{f x}$.

Note that, at zero incidence, the flow is axisymmetric, and the recirculation is a closed toroid. At an angle of attack, the flare shock is stronger on the windward side and weaker on the leeward side. If no circumferential communication, or crossflow, occurs, the streamwise recirculation should be larger on the windward side and smaller on the leeward side. However, the variation of shock strength, in addition to the imposed angle of attack, results in a strong crossflow which causes the flowfield to behave in the opposite manner. The crossflow sweeps much of low momentum fluid from the wind ward boundary layer to the leeward boundary layer. This leads to a forward movement of separation on the leeward side, with the process resulting in the thinning and strengthening of the windward boundary layer and thickening of the leeward side and an aft movement windward, as can be seen in the upstream rise of surface pressure and the plots of streamwise skin friction $C_{f x}$ in Fig. $9 \mathrm{~b}$. Moreover, as the angle of attack increases to $\alpha=8 \mathrm{deg}$, the lee-side crossflow separates, not only in the streamwise recirculation region (Fig. $9 \mathrm{a})$, but also in the ogive-cylinder portion ahead of the shockwave and boundary-layer interaction region. The experimental results show that the existence of lee-side separation, which now sweeps the low-momentum fluid away from the most leeward plane, $\phi=180 \mathrm{deg}$, thins the boundary layer and leads to a very small streamwise separation (Fig. 2) and a sharp pressure peak near the flare juncture (Fig. 7) around $\phi=180 \mathrm{deg}$. At $\alpha=8 \mathrm{deg}$, the calculated results from both the parabolized Navier-Stokes code and the timedependent code predict a substantially weaker crossflow separation, as compared to the experimental observation and, hence, fail to simulate the features enhanced by the crossflow separation observed by the experiment. This indicates a need for further improvement in the turbulence modeling.

Figure 10 shows pressures on the $(x-r)$ planes for the three meridional angles, $\phi=0,90$, and $180 \mathrm{deg}$. These pressure plots clearly show the variation of the static pressure field, which is first under a compression from the flare shock (various shock strengths) and is then followed by a strong expansion over the convex corner.

The three-dimensional, time-dependent program is coded in such a way as to permit treatment of a general body of revolution, i.e., $r_{b}=f(x)$. In the present numerical procedure, the program requires $0.00048 \mathrm{~s}$ per grid point per time step on a CDC 7600 . With a grid of $45 \times 34 \times 20$, it takes about $1.7 \mathrm{~h}$ of computation time for a converged steady solution. The option of solving the time-dependent, full 


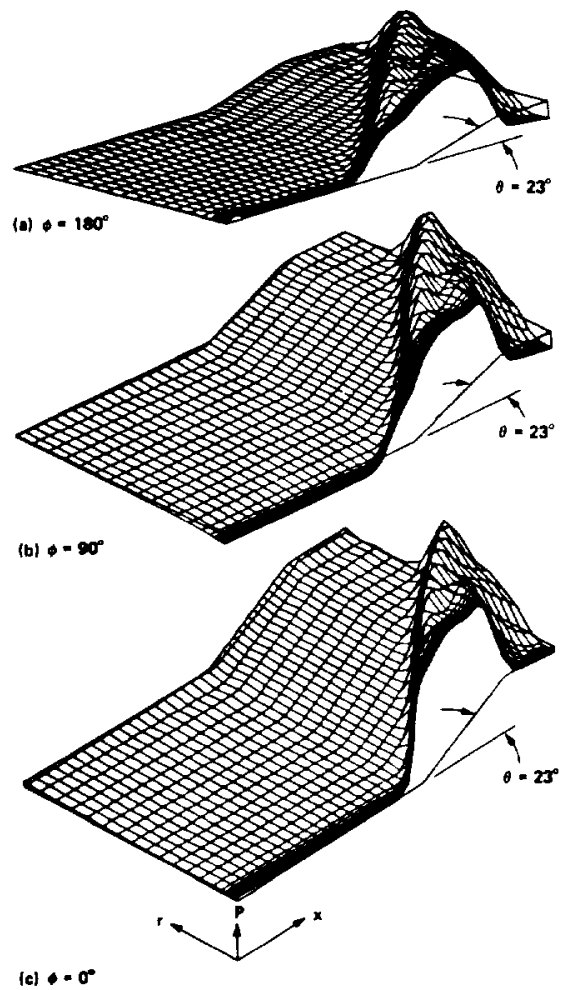

(c) $\cdots 0^{\circ}$

Fig. 10 Static pressure on $(x-r)$ planes at three circumferential angles for $\alpha=4$ deg.

Navier-Stokes equations is also coded. It requires about $25 \%$ more computation time and, for the present case, results from the thin-layer approximation and the complete Navier-Stokes equation agree within $4 \%$. Similar agreement is also experienced in the laminar flow case ${ }^{9}$ as well as in Ref. 14 . The parabolized technique takes about $14 \mathrm{~min}$ of computation time for marching a data plane consisting of a $40 \times 19$ grid 738 steps up to $x_{0}=6 D_{0}$, which is ahead of the interaction region. It takes about $1 \mathrm{~min}$ of CPU time to obtain a complete ogivecylinder-frustum supersonic inviscid solution $(24 \times 19 \mathrm{grid}$ and 340 steps).

\section{Concluding Remarks}

A supersonic turbulent flow over an ogive-cylinder-frustum at angles of attack $\alpha=0,4$, and 8 deg has been calculated numerically. The ogive-cylinder portion was solved efficiently by a parabolized Navier-Stokes technique. The shock-wave and boundary-layer interaction in the cylinder-frustum portion was then solved by the time-dependent, thin-layer approximation of the Navier-Stokes equations, with the upstream boundary conditions prescribed from the parabolized technique. Good agreement was obtained between computed and experimental results for surface pressure on the ogive-cylinder portion, and on the cylinder-flare portion at $\alpha=0$ and $4 \mathrm{deg}$. At $\alpha=8 \mathrm{deg}$, the agreement is still very good for the windward side. But the computed results failed to predict a sharp pressure peak near the cylinder-flare juncture. The results show that, while there is either no crossflow separation, or only a weak crossflow separation on the ogive-cylinder portion, the crossflow does effectively transfer low or "negative" momentum fluid to the leeward side and causes a small separation on the windward side with a large separation occurring on the leeward side. As angle of attack increases, the experimental results show that a strong crossflow separation convects the low-momentum fluid away from the leeward plane, thus leading to a small streamwise separation at $\phi=180 \mathrm{deg}$, the most leeward plane. The calculated results fail to predict a small streamwise separation at $\phi=180 \mathrm{deg}$ and indicates further improvement in the turbulence modeling is needed.

\section{References}

${ }^{1}$ Kutler, P., Warming, R. F., and Lomax, H., "Computation of Space Shuttle Flow Fields Using a Noncentered Finite-Difference Scheme," AIAA Journal, Vol. 11, Feb. 1973, pp. 196-204.

${ }^{2}$ Chyu, W. J. and Coe, C. F., unpublished data, 1967, and private communication.

${ }^{3}$ Lubard, S. C. and Helliwell, W. S. " Calculation of the Flow on a Cone at High Angle-of-Altack," AIAA Journal, Vol. 12, Feb. 1974, pp. 277-284.

${ }^{4}$ Lin, T. C. and Rubin, S. G., "Viscous Flow Over a Cone at Incidence, Part 2: Boundary Layer," Journal of Fluid Mechanics, Vol. 59, Pt. 3, 1973, pp. 593-620.

'Vigneron, Y. C., Rakich, J. V., and Tannehill, J. C., "Calculation of Supersonic Viscous Flow over Delta Wings with Sharp Subsonic Leading Edges," AIAA Paper 78-1137, June 1978.

6 McRae, D. S. and Hussaini, M. Y., "Numerical Simulation of Supersonic Cone Flow at High Angle of Attack," High Angle of Altack Aerodynamics, AGARD CP 247, Sandefjord, Norway, Oct. 1978, Paper 23.

'Schiff, L. B. and Steger, J. L., "Numerical Simulation of Steady Supersonic Viscous Flow." AIAA Journal, Vol. 18, Dec. 1980, pp. $1421-1430$.

"Schiff, L. B. and Sturek, W. B., "Numerical Simuiation of Steady Supersonic Flow over an Ogive-Cylinder-Boattail Body," AIAA Paper 80-0066, Jan. 1980.

${ }^{9}$ Hung, C. M., "Numerical Solution of Supersonic Laminar Flow Over an Inclined Body of Revolution," AIAA Journal, Vol. 18, Aug. 1980, pp. $921-928$.

${ }^{10}$ Beam, R. and Warming, R. F., "An Implicit Factored Scheme for the Compressible Navier-Stokes Equations," AIAA Journal, Vol. 16, April 1978, pp. 393-402.

"MacCormack, R. W. " An Efficient Numerical Method for Solving the Time-Dependent Compressible Navier-Stokes Equations at High Reynolds Number," NASA TM X-73, 129, July 1976.

${ }_{12}$ Baldwin, B. S. and Lomax, H., "Thin Layer Approximation and Algebraic Model for Separated Turbulent Flows," AlAA Paper 78257, Huntsville, Ala., Jan. 1978.

${ }^{13}$ Cebeci, T. and Smith. A.M.O., Analysis of Turbulent Boundary Layer, Academic Press, New York, 1974, p. 212.

${ }^{14}$ Hung, C. M. and Kurasaki, S. S." "Thin-Layer Approximation for Three-Dimensional Supersonic Corner Flows," AlAA Journal, Vol. 18, Dec. 1980, pp. 1544-1546. 